\title{
Una conversación con Michael Billig
}

\author{
J. MANUEL SABUCEDO * \\ Universidad de Santiago. Facultad de Psicología
}

El profesor Michael Billig es uno de los psicólogos sociales europeos actuales más interesantes. El Profesor Billig realizó sus estudios en la Universidad de Bristol, donde en el año 1972 presentó su tesis doctoral «Social Categorization and Intergroup Relations». Durante los años 1968 a 1972 fue Research Assistant de los Profesores H. Tajfel y C. Fraser, dedicándose especialmente al estudio de las relaciones intergrupo. Desde 1973 a 1984 ocupó el puesto de Lecturer en el departamento de Psicología de la Universidad de Birmingham. En el año 1985 pasa a desempeñar el puesto de Professor en el departamento de Social Sciences en la Universidad de Loughborough, cargo que ocupa actualmente.

Los primeros trabajos del Profesor Billig sobre categorización y relaciones intergrupo son bien conocidos por toda la comunidad de psicólogos sociales. Posteriormente, sus intereses, e incluso su orientación teórica y metodológica, parecen haber cambiado. En títulos como Ideology and Social Psychology (1982), Arguing and Thinking (1987), Ideological Dilemmas (1988), etc. (al final de la entrevista presentaremos una selección de los trabajos de M. Billig) podemos encontrar las líneas generales y los detalles de la aproximación retórica defendida por este autor.

Al margen de la valoración personal que se haga de los nuevos planteamientos teóricos del profesor Billig, lo que sin duda dependerá de las adscripciones teóricas y prácticas de socialización académica en las que cada uno de nosotros ha estado o está inmerso, lo que si parece fuera de toda duda, es la originalidad y la creatividad de sus propuestas. Sin duda, y como plantea Moscovici en uno de sus trabajos, uno de los mayores problemas de la psicología social es la compulsión a elaborar microteorías, sobre las que se desarrollan cientos de experimentos, y que después desaparecen sin saber muy bien por qué. Si estamos de acuerdo con ese diagnóstico de la situación, y pensamos que muchos lo estarán, los planteamiento de Billig deben ser recibidos como estimulantes cara al desarrollo de un mayor nivel de teorización en psicología social.

Pregunta: Profesor Billig, su departamento se caracteriza por tener una vocación interdisciplinar en la que confluyen áreas como psicología social, sociologia y administración y política social. Sin embargo, llama la atención que lo que podiamos llamar psicología general, asi como otras disciplinas de la psicologia no estén integradas en este departamento: ¿Cuál cree usted que debe ser la relación de la psicologia social con otras disciplinas cientificas, $y$ en concreto con la psicologia?

Respuesta: La psicología social debe ser considerada como una ciencia social. Es importante que los psicólogos sociales tengan un conocimiento amplio de otras ciencias sociales como sociología, antropología y ciencia po-

Agradecimientos: Agradezco a J. Francisco Morales sus sugerencias a la hora de elaborar el guión de esta entrevista. 
lítica. Creo que en el pasado la psicología tuvo demasiada influencia sobre la psicología social lo que se tradujo en que los psicólogos sociales prestasen muy poca atención a las ciencias sociales y la psicología individual fuese el principal marco de referencia.

Por otra parte, no creo que tenga ninguna utilidad el debatir si un problema debe ser tratado por la psicología social, la sociología, la antropología, etc. Lo que interesa es el estudio de las ideas y el análisis de la realidad, me es igual que a eso se le llame psicología social, sociología o antropología. ¿Para qué necesitamos las fronteras?

P.: Hace años, usted desempeñó un papel decisivo en la creación del paradigma de categorización intergrupo mínimo. ¿Qué importancia atribuye a ese paradigma en el desarrollo teórico de la psicología social en los últimos quince años?

$R .:$ Todos los paradigmas experimentales limitan el desarrollo teórico. Si ayudé a la creación de un paradigma, pido perdón por ello. Porque lo que ocurre con lo paradigmas es que la gente adopta una técnica experimental y empieza a aplicarla una y otra vez con muy ligeras modificaciones. $Y$ al proceder de esta forma, se deja de pensar en asuntos básicos y centrales, que es lo que posibilita el experimento original. Por ello, creo que ha habido demasiados experimentos que han usado el mismo diseño que aquellos trabajos originales sobre categorización intergrupo mínima.

P.: En el año 1976, usted publicó un libro sobre relaciones intergrupo, "Social psychology and Intergroup relations". ¿En su opinión, cuáles ban sido los principales cambios que se han producido en este área de trabajo, tanto en su vertiente de investigación como de teorización? En concreto, ¿cree que las expectativas iniciales han sido completamente satisfechas?

$R .:$ No lo sé. Para ser honesto debo decirle que no he seguido muy de cerca la investigación reciente sobre relaciones intergrupo o la psicología social de las relaciones intergrupo. Esto se debe en parte a que, como le comenté en la respuesta a la pregunta anterior, mucha de la investigación que se realiza parece estar destinada a matizar pequeños detalles de ideas básicas, muchas de las cuales han sido formuladas ya hace años por Henry Tajfel y Gordon Allport.

P.: Respecto a su trabajo académico, parece que ha cambiado desde una posición que es aceptada por la mayoría de psicólogos sociales a una más minoritaria. Me refiero a su trabajo inicial en grupos de investigación que utilizaban una metodologia experimental, y a las mayores simpatías que abora parece manifestar por las aproximaciones cualitativas. ¿Está de acuerdo con esta valoración?

$R .:$ No. No estoy de acuerdo en que la aproximación cualitativa sea menos aceptada, sobre todo en Gran Bretaña. Creo que entre las nuevas generaciones de psicólogos sociales en Gran Bretaña, hay actualmente una mayor preferencia por los métodos cualitativos que la que había hace quince años. $Y$ de hecho es posible que el número de postgraduados que en estos momentos realizan investigación en psicología social utilizando métodos cualitativos. Y si asumimos que en la psicología social europea la teoría de tante amplia.

P.: Pero, al margen de esto, ¿no cree que entre los psicólogos sociales en general, los métodos cualitativos son peor aceptados que los cuantitativos?

$R .:$ Los métodos cualitativos también son aceptados por aquellos que 
utilizan una metodología cuantitativa. Por ejemplo, si observamos el trabajo de Moscovici sobre representaciones sociales veremos que en algunos de sus escritos hay sugerencias claras de que deberían utilizarse métodos cualitativos. Y si asumimos que en la psicología social europea la teoría de las representaciones sociales es una posición aceptada, entonces también hay que afirmar que la metodología cualitativa es una metodología aceptada. Sin embargo, muchos editores, por razones que no logro entender, no siempre asumen esto, y es evidente la dificultad de publicar trabajos cualitativos en revistas como European Journal of Social Psychology o British Journal of Social Psychology. Me gustaría creer que esta situación está cambiando, pero sospecho que actualmente la mayoría de revistas no representan de modo adecuado el pensamiento de los psicólogos sociales en Gran Bretaña y en Europa. te?

P.: Pero, ¿̇por qué abandonó usted la que llamábamos posición dominan-

$R .:$ Mi primer trabajo con metodología cualitativa, fue un estudio sobre el fascismo, donde incluía entrevistas con miembros de ese grupo político y analizaba la propaganda del partido fascista. Obviamente, un paradigma experimental no era apropiado para descubrir cómo pensaban los fascistas. En esa investigación realicé algún análisis cuantitativo de la ideología, realicé algún análisis de contenido de su propaganda impresa, y para mí resultó muy claro que el análisis cuantitativo no decía realmente nada sobre muchos de los temas subyacentes de la ideología. De hecho, el análisis cuantitativo sólo nos contaba lo que el propagandista quería que el lector casual pensara, pero sus mensajes tenían significados encubiertos. Mensajes que eran dirigidos a aquellos que entendían la dinámica y el significado subyacente de la ideología. Y para probar esto necesité recurrir al análisis cualitativo. También cuando hablaba con los miembros del grupo fascista, estaba mucho más interesado en el significado de lo que ellos decían que en cuántas veces usaban una palabra determinada.

$P .:$ ¿ No cree que la subjetividad del investigador puede resultar un problema importante para la metodología cualitativa?

$R .:$ Todos los métodos tienen problemas. Los métodos cualitativos también los tienen, pero no creo que la subjetividad del investigador sea un problema particular que afecte exclusivamente a los métodos cualitativos. Si pensamos en los métodos cuantitativos, ciertos procedimientos son bastante claros una vez decidida la prueba estadística a utilizar. Pero una vez aplicada la prueba estadística, tenemos que interpretar los resultados. Todos los métodos cuantitativos incluyen interpretación, al igual que los cualitativos. De hecho, sin interpretación las ciencias sociales serían increíblemente aburridas.

P.: Me gustaría conocer su opinión sobre el estado actual de la metodologia dominante en psicología social. ¿Cree que ha habido un avance sustancial respecto al periodo en que se elaboraron teorias tan importantes como las del cambio de actitudes y atribución?

$R .:$ Algunas de las ideas que sostenían las investigaciones sobre las teorías de las actitudes y atribución pretendieron elaborar un tipo particular de teoría. Creo que esa investigación no ha tenido éxito en conseguir ese objetivo. Además debo decir que esto me satisface, porque si esos objetivos se hubiesen conseguido darían lugar a teorías que posibilitarían a los 
investigadores predecir y controlar las respuestas de las personas que reciben los mensajes. Si la investigación hubiese resultado exitosa y se encontrara que se podía predecir y controlar las respuestas de los receptores del mensaje, sería una panacea para los propagandistas y políticos y estaríamos moviéndonos hacia un estado totalitario, manipulado y controlado. Afortunadamente, aunque esto sea algo más que suerte, la investigación no ha tenido éxito y no se ha desarrollado por ese camino.

P.: Permitame que insista en la cuestión del conocimiento actual de la psicología social sobre el tema de las actitudes. ¿Cree usted que boy nuestro conocimiento sobre las actitudes y el cambio de actitudes es mayor que el que tenian los viejos retóricos?

$R .:$ Esa pregunta no puede responderse simplemente con un sí o un no. La cuestión es mucho más complicada porque se refiere a lo que nosotros entendemos por conocimiento y las relaciones de ese conocimiento con los contextos sociales. A pesar de esto, voy a tratar de responder a su cuestión. Si estuviese encerrado en prisión y me dijesen que sólo podía tener un libro y que tenía que ser un libro sobre actitudes, preferiría el trabajo de Aristóteles sobre retórica que cualquier volumen o todos los volúmenes del Journal of Personality and Social Psychology.

$P_{.:}$¿Qué opinión le merece el actual nivel de teorización en psicologia social?

$R .:$ Creo que hay problemas con el nivel de teorización. Bastantes constructos teóricos o teorías han sido tratadas de modo muy erróneo y equivocado. Ha habido una enorme cantidad de desarrollo conceptual confuso. No tenemos más que fijarnos en la teoría de la atribución para comprobar las confusiones conceptuales que frecuentemente han sido cometidas por los investigadores al usar conceptos claves de una forma vaga y hueca y frecuentemente ocultos detrás de una masa impenetrable de jerga aparatosamente resonante.

P.: ¿Qué solución apuntaría usted para solventar ese problema?

$R$.: No hay una solución fácil en el sentido de decir que lo que habría que hacer es esto o aquello. El desarrollo teórico en las ciencias sociales no se produce de una forma tan sencilla como esa. Lo que diría es que para incrementar el nivel de conciencia teórica entre aquellas personas que se dedican a la investigación psicosocial, sería necesario que éstas tuviesen un abanico de lecturas mucho más amplio que el que tienen actualmente. Deberían leer seriamente filosofía, sociología y antropología.

P.: Su interés en los últimos años se ba centrado en el estudio de la ideologia. Hay personas que piensan que cada disciplina debe estudiar las cuestiones que le son propias y no otras. De este modo, la ideología debería ser objeto de tratamiento de la ciencia política y no de la psicología social. ¿Qué piensa usted de esto?

$R .:$ Realmente mi interés por la ideología no se limita a estos últimos años. Como usted sabe, la ideologia era un tema que ya estaba presente en el libro que mencionó con anterioridad, Social Psychology and Intergroup Relations. Pero respondiendo a su pregunta, creo que las fronteras en la vida intelectual son peligrosas. Siempre que alguien diga «Oh, usted no debería estudiar esto, usted no debería leer esto, esto no es parte de su provincia», está deshonrando al trabajo intelectual. Débemos abordar cualquier problema en el que estemos interesados e ir tan lejos como podamos. 
P.: ¿Cómo puede la psicología social contribuir al estudio de la ideología?

$R$.: ¿Puedo darle la vuelta a la cuestión y plantear qué tipo de cuestiones asociadas con los problemas de la ideología son psicosociales? Este tipo de cuestiones son, por ejemplo, ¿cómo la ideología afecta al modo en que piensan las personas? ¿De qué modo piensa la gente en su vida ordinaria? ¿Usan conceptos y significados que tienen una larga historia ideológica? ¿Cómo aprenden los niños estos conceptos ideológicos?, y ¿Cómo al usar los conceptos alteran el patrón de la ideología? Estas son cuestiones centrales al estudio de la ideología y son cuestiones, creo, psicosociales o que tienen una dimensión psicosocial. Pero al estudiarlas desde una perspectiva psicosocial también se necesita estudiar la naturaleza de la ideología, el papel de la ideología en la sociedad y la historia de la ideología. Por eso, como ya le comenté, el estudio de la psicología social debería abrirse al estudio de otras ciencias sociales.

P.: ¿Puede el estudio de la ideología ayudar al desarrollo de la psicología social?

$R$.: Por supuesto. Todo conocimiento puede ayudar al desarrollo de cualquier disciplina intelectual. Si por el estudio de la ideología entendemos la obtención de conocimiento acerca de dónde proceden las ideas y cuáles son sus funciones en la sociedad, obviamente el estudio de la ideología puede ayudar al desarrollo de la psicología social. Permítame un ejemplo. $\mathrm{Al}$ igual que otros psicólogos sociales actuales, siento una gran admiración por el trabajo de Vygotski y por sus estudios sobre el uso y la adquisición del conocimiento en los niños. Y las teorías de Vygotski se basan en gran medida en las teorías de la ideología y la construcción social del pensamiento de Marx. Creo que éste es un buen ejemplo de cómo el conocimiento sobre el concepto de ideología y los importantes trabajos de Marx sobre la ideología dieron forma a una importante teoría psicológica.

P.: Sus planteamientos teóricos subrayan la naturaleza social y el carácter dialéctico y retórico del pensamiento humano. En su último libro «Ideological Dilemmas» afirma que las ideas de prejuicio y tolerancia existen como ideas de sentido común dentro de la sociedad. De acuerdo con esto la solución al problema del prejuicio se halla en el nivel social y no en el individual o grupal. ¿Cuál sería entonces la contribución de la psicología social a la solución de éste y otros problemas sociales?

$R .:$ La cuestión es cómo pueden los científicos sociales ayudar a la solución de problemas sociales. Para muchos problemas sociales, y el prejuicio es uno de ellos, no hay una solución simple. Si la hubiese la gente la conocería desde hace ya mucho tiempo. Es irreal esperar que los científicos sociales digan "Aquí está la solución al problema del prejuicio, vayan y aplíquenla».

Pero los académicos tienen una gran responsabilidad en los problemas sociales y en el estudio de los mismos, especialmente en el del prejuicio. La razón es simple. El pensamiento académico no tiene lugar en un medio aislado del resto de la sociedad. Las ideas de los académicos influyen en la sociedad y, frecuentemente, esas ideas se convierten en parte del problema. Si observamos las opiniones del prejuicio y de las diferencias raciales, comprobaremos que frecuentemente éstas proceden de opiniones de pensadores, de científicos sociales, de filósofos, que se han difundido ampliamente en la sociedad convirtiéndose, entonces, en parte del problema. Por tanto, 
es responsabilidad de todos los académicos pensar y tratar sus problemas lo más clara y honestamente que puedan, porque sus ideas pueden introducirse dentro de la sociedad y llegar a ser usadas por la gente ordinaria. Eso significa que una razón para estudiar ciencias sociales es porque uno piensa que las ideas que está formulando son mejores o más verdaderas que otras. Y quiere que sus ideas, en las que cree, salgan al exterior y lleguen a ser usadas fuera de la academia por la gente de la calle. Y que esa gente las acepte antes que a aquellas ideas a las que combatimos.

P.: Entonces, ¿deberiamos olvidarnos de muchas de las. soluciones brindadas por la psicología social para la solución del tema del prejuicio, especialmente aquéllas con un carácter más marcadamente individual, y centrarnos en el discurso social?

$R .:$ No creo que esos dos tipos de actividades sean necesariamente contrapuestos. En mi trabajo sobre los fascistas, examiné las razones que podían conducir a diferentes personas a formar parte de partidos fascistas. Si el conocimiento psicosocial sobre esos temas puede ser utilizado para persuadir a ese tipo de personas para que abandonen esas posiciones, sería beneficioso. Pero, al mismo tiempo no creo que la amenaza o los peligros del fascismo y racismo desapareciesen si lográsemos persuadir a una o dos personas de lo erróneo de sus posturas políticas.

Por otra parte, hay que señalar que ha habido trabajos psicosociales en este campo realmente importantes. Estoy pensando en el libro de Myrdal «The American Dilemma». Esta es una pieza soberbia de ciencia social. Es un libro muy valioso y creo que desde su publicación ha tenido un efecto muy beneficioso sobre el modo en que los americanos piensan sobre las relaciones interraciales.

P.: En una respuesta anterior, usted se refirió al papel que en estas cuestiones desempeñan los científicos sociales. ¿Cree usted que el científico social debe comprometerse con su realidad y tratar de modificarla?

$R$.: Sí. No puede ser de otro modo. El científico social vive en el mundo. La actividad científico social es una actividad social.

Actualmente, creo que en Gran Bretaña existe un gran peligro de que las ciencias sociales sean corrompidas al tratar de conseguir las ayudas gubernamentales para la investigación. Los científicos sociales no están tratando de definir sus propios temas y problemas intelectuales, sino que intentan dedicarse a aquellas tareas que le interesan al gobierno. Si los científicos sociales no reflexionan sobre lo que están haciendo, corren el riesgo de corromper tanto sus propias disciplinas como sus vidas intelectuales.

P.: Una última cuestión. Al margen de sus trabajos más teóricos sobre la aproximación retórica a la psicologia social, ¿̇en qué temas está usted actualmente trabajancio?

$R$.: Sigo interesado en problemąs relacionados con la ideología. Actualmente estoy estudiando cómo las familias británicas hablan de la familia real. Este discurso en el que las familias hablan y discuten entre ellos sobre la familia real es ideológicamente muy rico. Todo el mundo en Gran Bretaña conoce a la familia real, todos parecen tener opiniones sobre lo que la familia real y sus miembros deberían hacer, y cuando hablan de ellos aparecen concepciones sobre el privilegio, la igualdad, la naturaleza de la familia, la naturaleza de las relaciones humanas. Así en su discurso sobre la realeza, ellos expresan valores socio-ideológicos muy importantes. 


\section{Selección de trabajos de Michael Billig}

Libros

BILlig, M. (1976). Social Psychology and Intergroup Relations. London: Academic Press.

BILlug, M. (1978). Fascists: A Social Psychology View of the National Front. London: Academic Press.

BILlIG, M. (1981). L'Intermationale Raciste: de la psychologie a la science des races. Paris: Mampero (edición alemana: Die Rassistische Internationale. Frankfurt: Neue Kritik).

BILlig, M. (1982). Ideology and Social Psychology. Oxford: Basil Blackwell.

BILLIG, M. (1987). Arguing and Thinking: A Rhetorical Approach to Social Psychology. Cambridge University Press.

Billig, M.; Condor, S.; Edwards, D.; Gane, M., y RADleY, A. R. (1989). Ideological Dilemmas in Everyday Thinking. London: Sage Publications.

\section{Capitulos}

BILlig, M. (1984). Political Ideology: Social psychological aspects. En Tajfel, H. (Ed.). The Social Dimension: European Developments in Social Psychology. Cambridge: Cambridge University Press.

Bıllig, M. (1984). Racisme, prejuges et discrimination. En Moscovici, S. (Ed.). Psychologie Sociale. Paris: Presses Universitaires de France.

Billig, M. (1986). Political Psychology and social psychological theory. En Brouwer et al. (Eds.). Political Psychology in the Netherlands. Amsterdam: Mola Russa.

BiLlig, M. (1986). Very ordinary life and young conservatives. En Beloff, H. (Ed.). Getting Into Life. London: Methuen.

BILLIG, M. (1987). Anti-semitic themes and the British far left: some social-psychological observations on indirect aspects of the conspiracy tradition. En Graumann, C. F., y Moscovici, S. (Eds.). Changing Conceptions of Conspiracy. Springer Verlag: New York.

Billig, M. (1988). Methodology and scholarship in understanding ideological explanation. En Antaki, C. (Ed.). Analyzing Lay Explanation. Sage: London.

Billig, M. (in press). Collective memory, ideology and the British Royal Family. En Middleton; D., y Edwards, D. (Eds.). Collective Remembering. Sage: London.

BILLIG, M. (in press). Studying the thinking society: social representations, rhetoric and attitudes. en Beakwell, G., y Canter, D. (Eds.). Empirical Approaches to Social Representations. Oxford University Press.

Billig, M. (in press). Rhetoric of Social Psychology. En Parker, I., y Shotter, J. (Eds.). Deconstructing Social Psychology. Routledge and Kegan Paul: London.

Billig, M. (in press). Foreword. En Windisch, U. (Ed). Speech and Reasoning in Everyday Life. Cambridge University Press: Cambridge.

Articulos

Fraser, C.; Gouge, C., y Billig, M. (1971). Risky shifts, cautious shifts and group polarization. European Journal of Social Psychology 1, 7-30.

BILLIG, M., y TAJFEL, H. (1973). Social categorization and similarity in intergroup behaviour. European Journal of Social Psychology 3, 27-52.

TAJFEL, H., y BILLIG, M. (1974). Familiarity and categorization in intergroup behaviour. $E u$ ropean Journal of Social Psychology 10, 159-170.

BILLIG, M. (1977). The new social psychology and «fascism». European Journal of Social Psychology.

Billig, M., y Cochrane, R. (1981). The National Front and youth. Patterns of Prejudice 15, 3-15.

BILLIG, M. (1982). The origins of race psychology. I. Patterns of Prejudice 16, 3-16.

Billig, M. (1985). Prejudice, categorization and particularization: a rhetorical approach. $E u-$ ropean Joumal of Social Psychology 15, 79-103.

BILLIG, M. (1988). Rhetorical and historical aspects of attitudes: the case of the British Monarchy. Philosophical Psychology 1, 83-104.

BILLIG, M. (1988). Social representation, anchoring and objectification: a rhetorical analysis. Social Behaviour 3, 1-16.

BILLIG, M. (1988). The notion of prejudice: some rhetorical and ideological aspects. Text 8 , 191-217.

BILLIG, M. (1988). Rhetoric of the conspiracy theory: arguments in National Front propaganda. Patterns of Prejudice 22, 23-34.

BILLIG, M. (in press). The argumentative nature of holding strong views: a case study. European Journal of Social Psychology.

BILlig, M. (in press). Destructive forces: neo-nazis and the Pevisionist. Patterns of prejudice.

Billig, M. (in press). Psychology, rhetoric and cognition. Journal of the history of Human Sciences. 